\title{
Electrical stimulation: then and now. Applications and limitations
}

\author{
DOI: https://doi.org/10.5114/pq.2021.11098
}

\author{
Aneta Demidas ${ }^{\oplus}$ \\ Department of Massage and Physical Therapy, Faculty of Physiotherapy, Wroclaw University of Health and Sport Sciences, \\ Wroclaw, Poland
}

\section{Abstract}

Electrical stimulation is a branch of physical therapy that applies low and medium frequency currents to stimulate the human body. The effects of electrical impulses on human beings have been observed since antiquity. The development of research in that scope commenced in the 18th century, the most important turning point, and is now continuing. The purpose of this narrative study is to present electrical stimulation in terms of its progressing development and to draw attention to its significant role in the therapeutic processes utilized in numerous medical specializations and comprehensive strength and endurance training of healthy people. The notions 'electrical stimulation' and 'electrotherapy,' as well as differences between electric current applications are explained. The most critical moments in the history of electrical stimulation development are highlighted. Recent research is presented, exhibiting the important role of electrical stimulation both in the therapeutic process and in strength and endurance training. This paper contains the most significant aspects of contemporary application of electrical stimulation, as well as recommendations and limitations for current usage in such areas as whole-body electrostimulation, urinary incontinence, pelvic floor muscle rehabilitation, rehabilitation after anterior cruciate ligament surgery, endurance training, and improvement of physical strength and appearance.

Key words: electrical stimulation, electrotherapy, history of electrotherapy, types of electrical stimulation, muscle pelvic floor, whole-body electrostimulation

\section{Introduction}

The term 'electrical stimulation' remains somehow unclear and unexplained. Physiotherapists have doubts both as to the parameters of the currents used for electrical stimulation and their correct application and as to which electrotherapeutic treatments can be called electrical stimulation and which of them - electrotherapy. The issue is even more important that the recent years have observed a rise in the applications of electrical stimulation in different medical disciplines which had not taken it into account as a tool supporting therapy or had used it to a small degree. Such disciplines include urology, otolaryngology, gynaecology, and neurology, which rarely apply electrical stimulation as a tool supporting therapy or even solving the patients' problems in some cases of nerve paralysis. The benefits of electrical stimulation are most noticeable in orthopaedics and sports medicine. In sports, electrical stimulation as a procedure supporting regular training to increase muscle strength and endurance is nothing innovative nowadays [1-3]. Current fitness facilities also offer training with the use of stimulation with low and medium frequency currents. In this kind of training, one can observe a comprehensive simultaneous effect on multiple muscles due to the application of several or over a dozen electrodes to the body at the same time.

Applying electrical stimulation in aesthetic medicine as a non-surgical facelift procedure constitutes a real innovation. Electrostimulation is an important tool for therapeutic rehabilitation, as well as for muscle strength and condition improvement in personal training and sports. Therefore, some issues should be clarified and systematized.

\section{Is there any difference between electrotherapy and electrical stimulation?}

Actually, each application of current for therapeutic purposes can be named electrotherapy or electrical stimulation as each elicits stimulating activity upon the body and gives results ranging from pain reduction to stimulation of muscle contraction.

Therapy with current application and its parameters are chosen in accordance with 3 main types of body response to electrostimulation: sensory, motor, and nociceptive pain, so the therapy works in the mentioned areas [4]. The point is not to indicate the difference between electrotherapy and electrostimulation, but to appropriately adjust the current and its parameters for the needs of the patient's or athlete's therapeutic rehabilitation, therapy or strengthening training. As for the parameters, the following should be taken into account: current/waveform, current amplitude, duration, frequency, modulation, on and off times/cycling, ramp-up and -down times, total treatment times, and electrode set-up [5, 6].

There are 3 types of most commonly used currents: direct current, pulsatile/pulsed current, and alternating current. For electrostimulation purposes, pulsatile/pulsed current is widely adopted, such as in neuromuscular electrical stimulation (NMES), microcurrent electrical stimulation (MES), functional electrical stimulation (FES), transcutaneous electrical nerve stimulation (TENS), percutaneous electrical nerve stimulation (PENS).

TENS is a method of pain relief and most often to treat muscle and joint problems [7]. It is used for neuromuscular re-education and treatment of muscle atrophy [8]. NMES is mostly applied in sports medicine for muscle strengthening, as well as maintenance of muscle mass and strength [9]. FES

Correspondence address: Aneta Demidas, Department of Massage and Physical Therapy, Faculty of Physiotherapy, Wroclaw University of Health and Sport Sciences, al. I.J. Paderewskiego 35, 51-612 Wroclaw, Poland, e-mail: aneta.demidas@awf.wroc.pl, https://orcid.org/0000-0002-8712-2312 
is applied to paralyzed or weakened muscles to restore or improve their function [10]. In turn, MES utilizes low-intensity current similar to the body's own natural current, which enhances endogenous healing effects [11].

The use of these types of currents in accordance with the patient's needs can also be named electrotherapy because by improving the functional state of muscles, it improves the patient's condition.

Recently, an interesting solution in electrotherapy has been the usage of direct current, so-called percutaneous microelectrolysis (MEP). In MEP procedures, the direct current is very low (up to $0.96 \mathrm{~mA}$ ), and it is delivered to the patient's body through a negative polarity acupuncture needle. MEP induces a controlled local inflammatory process that stimulates tissue repair and is accompanied by an analgesic effect. Research concerning MEP demonstrates positive effects, inter alia, decreasing pain in myofascial trigger points [12].

In conclusion, it should be emphasized that the essence of the matter is physiotherapists' awareness and knowledge regarding the methods used, their compliance with guidelines, their ability to diagnose disorders and operate electrotherapy and electrostimulation devices, their manners and ethical principles that guide them in work. These factors eventually contribute to achieving a therapeutic/sports goal [6].

It is valuable to follow the achievements, procedures, policies, and guidelines of such institutions in the field of physical therapy as the World Confederation for Physical Therapy, International Society for Electrophysical Agents in Physical Therapy, and American Physical Therapy Association [13].

\section{Some history: about the beginnings}

Stimulation with current is viewed as one of the oldest methods used in physiotherapy, of which electrical stimulation has a relatively long, 150-year history. The foundations of electrical stimulation procedures were laid in each subsequent period, beginning from ancient Rome and Greece.

\section{Antiquity}

As early as in $420 \mathrm{BC}$, Hippocrates noticed the properties of electric fish and applied them to treat diseases. Torpedo fish were used for that purpose. The body of the fish is like a disc, which translates into its big surface. On its sides, that fish has 2 organs producing electric voltage. The biggest specimen may produce a voltage up to $200 \mathrm{~V}$. The procedures of electrical stimulations of the human body with the electric properties of fish were carried out with the use of unique tubs filled with the said fish. The patients put their legs into the tube, and the treatment was administered. In ancient Greece, also electric eels put into the tubes for feet soaking were applied for treatment. The reports concerning the use of properties of fish producing electric discharges in therapy date back to $46 \mathrm{AD}$. It was recorded that Scribonius Largus placed electric fish on painful body parts such as the head, aching tooth, or spine.

\section{Modern times}

In the $18^{\text {th }}$ century, when electric phenomena were discovered and studied, therapies with electric current became trendy throughout Europe. In 1743, Johann Gottlob Krüger, a Professor at the university in Halle, commenced applying electrical therapy in the stimulation of human muscles and other organs. He gave lectures on medicine as well as physics and electricity. It was the time when Christian Gottlieb Kratzenstein treated patients with paralysis symptoms with the use of the so-called static electricity. In 1746, he published a treatise on electrotherapy entitled Theoria electricitatis more geometrico explicata. He constructed a machine for electrization for therapeutic purposes. The procedure involving its use was called 'franclinization' later on, after the name of Benjamin Franklin (1706-1790), a statesman, scientist, inventor, electricity researcher, creator of the notion of positive and negative electricity, the inventor of the lightning conductor.

Luigi Galvani, an Italian physician, physicist, biologist, and philosopher, discovered in 1780, together with his wife, that the muscles of a dead frog's legs twitched when struck by an electrical spark. He was the first to describe the contraction of muscles and nerves caused by the passing of electric current.

Slightly later, in 1799, Alessandro Volta, an Italian physicist and chemist, continued Galvani's experiments and demonstrated that electric current was generated when 2 different metals were connected with an electric conductor and separated with a fabric saturated with a solution of acid or salt. On such grounds, Volta invented the first electric cell, which was the first artificial source of electricity used by man.

A continued improvement and expansions of the scope of applied techniques were observed since about 1850. Emil du Bois-Reymond was the first one to carry out stimulation of muscles and nerves with the use of faradic and galvanic current. In 1851, as a result of his observation, he recorded action currents generated during muscle contraction and formulated the law of contraction.

The turn of the $19^{\text {th }}$ and $20^{\text {th }}$ centuries was marked by the development of specialized facilities where direct current and faradic, i.e. interrupted current were used, especially hydroelectric baths. Direct current was also applied to strengthen the muscles and excite vascular nerves to accelerate blood circulation, while faradic current was mainly utilized to treat paralysis.

In the interwar period, Bergoni's currents were used to support slimming down, and Leduc currents were applied to treat paralysis, muscular atrophies, neuralgia, and obesity.

In 1934, in France, Le Go introduced a new type of current consisting of triangular pulses, which caused a stronger contraction in paralyzed or denervated muscles than in healthy ones.

In 1941, Bauwens used square pulses to determine the $\mathrm{i} / \mathrm{t}$ curve, which was the basis for determining the value of chronaxie $[8,14,15]$.

After World War II, a significant contribution to the development of electrical stimulation was made by Bobechko and Kotz. Bobechko introduced FES. He implanted electrodes into the paraspinal muscles to treat scoliosis.

In 1977, Yakov Kotz, a Russian sports physician, applied average alternating frequency current to stimulate correctly innervated muscles in athletes, Russian astronauts, and patients after amputations. Kotz current has been successfully used up to this day [16].

\section{Contemporary applications of electrical stimulation}

At present, treatment with electrical stimulation is a therapeutic solution in many diseases associated with incorrect muscle tension or neuromuscular paralysis; it is also applied in strength and endurance training of healthy muscles.

\section{Electrical stimulation for urinary incontinence}

Urinary incontinence is a common health problem affecting the quality of life of nearly 420 million people, both women and men. Pelvic floor muscle training and other physiother- 
apy techniques are essential in non-surgical treatment of urinary incontinence, bringing about good therapeutic results. It is estimated that about $30-40 \%$ of women without urinary incontinence dysfunction and about $70 \%$ of those with a pelvic floor dysfunction are unable to perform a correct pelvic floor muscle contraction; therefore, it is crucial to implement physiotherapeutic techniques aimed at early activation of pelvic floor muscles. At present, urinary incontinence physiotherapy is focused on electrical stimulation of pelvic floor muscles and the cooperation of such muscles with synergistic muscles, diaphragm, as well as education in correcting improper everyday habits for better pelvic organ support and continence [17].

The goal of the research performed by Jha et al. [18] was to evaluate the clinical effectiveness of standard pelvic floor muscle training combined with electrical stimulation of muscles compared with pelvic floor muscle training alone in women with urinary incontinence and sexual dysfunction. Overall, 114 women were examined. Physiotherapy turned out beneficial to improving overall sexual function. Electrical stimulation of muscles undergoing the therapy provided greater benefits in the scope of listed dysfunctions than pelvic floor muscle training alone.

Subsequent clinical research showed that electrical stimulation combined with pelvic floor muscle training could be applied in the effective treatment of postpartum urinary incontinence [19].

The influence of NMES on urinary incontinence was also examined in patients with urinary incontinence after stroke. A total of 82 participants were randomly assigned to 2 groups that received NMES therapy or sham NMES for 10 weeks. The primary efficacy endpoints were measured by urodynamic values and the Overactive Bladder Symptom Score. The secondary efficacy endpoints were assessed with the International Consultation on Incontinence Questionnaire - short form (ICIQ-SF), Barthel Index scale, and adverse events. All outcomes were evaluated at baseline and the end of 10 weeks of treatment. After 10 weeks of treatment, the patients receiving the NMES therapy demonstrated better efficacy in the primary endpoints of urodynamic values $(p<0.01)$ and the Overactive Bladder Symptom Score $(p<0.01)$, as well as in the secondary endpoints of ICIQ-SF $(p<0.01)$ and Barthel Index $(p<0.01)$ in comparison with patients who underwent sham NMES. No adverse events were recorded in either group [20].

\section{Electrical stimulation for pelvic floor muscle rehabilitation}

The goal of a study performed by Pastore et al. [21] was to assess the long-term results of pelvic floor muscle rehabilitation in males suffering from lifelong premature ejaculation diagnosed on the basis of intravaginal ejaculatory latency time (IELT) and the self-report Premature Ejaculation Diagnostic Tool (PEDT). In this research, a total of 154 participants were retrospectively reviewed, of whom $122 \mathrm{com}$ pleted the training protocol. At baseline, all participants presented with an IELT $\leq 60$ s and PEDT $>11$. The 12-week pelvic floor muscle rehabilitation programme included physiokinesiotherapy treatment, electrostimulation, and biofeedback, with 3 sessions per week, 20 minutes for each component at each session. The intervention effectiveness was evaluated by comparing the change in the geometric mean of IELT and PEDT values from baseline at 3, 6, and 12 months during the intervention, and at 24 and 36 months after the intervention. From among the 122 participants who completed the pelvic floor muscle rehabilitation, 111 gained control over their ejaculation reflex, with IELT $=161.6 \mathrm{~s}$ and PEDT $=2.3$ at the 12-week endpoint of intervention, which constitutes an increase from baseline of $40.4 \mathrm{~s}$ and 17.0 points, respectively $(p<0.0001)$. Among the 95 participants who completed a 36 -month follow-up, $64 \%$ and $56 \%$ retained satisfactory ejaculation control 24 and 36 months after the intervention, respectively.

La Pera [22] examined 78 patients with premature ejaculation. The goal of the research was to assess the cure rate in patients who underwent treatment involving: (1) awareness of pelvic floor muscles; (2) learning the timing of execution and maintenance of pelvic floor muscles contraction during the sensation of the pre-orgasmic phase; (3) pelvic floor rehabilitation: biofeedback, pelvic exercises, and electrical stimulation. The patients were first informed of the role of the pelvic floor. They were taught to carry out the execution and maintenance of pelvic floor muscles contraction during the sensation of the pre-orgasmic phase to control the ejaculatory reflex. In order to improve the awareness, tone, and endurance of the pelvic floor muscles, the participants were treated with pelvic floor rehabilitation consisting mainly in biofeedback, pelvic exercises, and, in some cases, electrical stimulation. The training was carried out for a period of about 2-6 months, with an average of 2-5 visits per cycle. As a result of the aforementioned therapies, $54 \%$ of patients who completed the training were cured from premature ejaculation and learned over time to postpone the ejaculation reflex. The best results were obtained mainly in patients aged below 35 years, where the cure rate was $65 \%$. No side effects were observed.

Laurienzo et al. [23] investigated the effects of electrical stimulation and pelvic floor muscle training on the strength of pelvic floor muscles, urinary incontinence, and erectile function in men with prostate cancer. They examined 123 men after radical prostatectomy. The patients were randomized into 3 groups: (1) a group performing pelvic floor muscle exercises; (2) a group receiving electrical stimulation of anus twice a week for 7 weeks and performing pelvic floor muscle exercises; (3) a control group. The primary outcome assessment was based on the measurement of pelvic floor muscle strength recovery in each group. The data were obtained preoperatively and after 1,3 , and 6 months after surgery. The greater urinary leakage and pelvic floor muscle weakness in the first month compared with the pre-treatment state improved after 3 and 6 months after surgery, without more significant differences among the groups.

The systematic review and meta-analysis performed by Kannan et al. [24] concerned the comparison of the effectiveness of pelvic floor muscle training alone and in combination with biofeedback, electrical stimulation, or both to control for urinary incontinence in men after prostatectomy. The analysis of numerous studies comprising a previous systematic review demonstrated that the most effective combination was the one that involved pelvic floor muscle training with electrical stimulation and biofeedback. The effect of muscle training and biofeedback alone on urinary incontinence turned out to be uncertain, similarly to pelvic floor muscle training alone.

\section{Electrical stimulation after anterior cruciate ligament surgery}

An example of the application of electrical stimulation is its use after anterior cruciate ligament (ACL) surgery. Reduced ability to contract quadriceps is often found immediately after the surgery. It can lead to muscle atrophy and decreased function. NMES may be a valuable intervention to ameliorate 
these deficits following ACL surgery. The goal of a review by Hauger et al. [25] was to determine whether NMES combined with standard physiotherapy was superior to standard physiotherapy alone in improving quadriceps strength or physical function after ACL surgery. The conclusion formulated as a result of the review was explicitly positive for neuromuscular stimulation. NMES, as a procedure additional to standard physiotherapy, significantly influenced the improvement of quadriceps strength and physical function in the early postoperative period compared with standard physiotherapy alone.

NMES is also helpful after ACL reconstruction. It was proved that it reduced quadriceps activation failure and, when combined with eccentric exercises, decreased the atrophy and increased the regeneration and strength of quadriceps after ACL reconstruction [9].

\section{Whole-body electrostimulation}

Recently, the combination of strength training with electrical stimulation of the whole body has been investigated. The research by Lopes Evangelista et al. [26] shows that 8 weeks of strength training combined with whole-body electrical muscle stimulation (WB-EMS) increased the morphofunctional parameters, especially the strength growth percentage of the examined muscles, in comparison with performing the strength training alone.

WB-EMS administration has its restrictions and may cause adverse reactions. Therefore, it is necessary to perform research which would define safe parameters of such stimulation and identify the adverse reactions. A literature search in PubMed revealed 11 trials, 3 cohort studies, and 7 case reports. Enormous creatine kinase elevations were reported in healthy volunteers. Creatine kinase levels were not tested in randomized studies, but several patients discontinued WB-EMS because of 'muscular discomfort'. The contraindications for WB-EMS are not clearly defined. There were 9 cases of rhabdomyolysis diagnosed after the intervention, especially after the first application. It is necessary to increase the safety of WB-EMS. Patients with a history of rhabdomyolysis should not undergo WB-EMS, and those experiencing rhabdomyolysis should be neurologically investigated. Since the value of WB-EMS as an alternative to physical exercises is uncertain, there is a need to prove or disprove its benefits [27].

NMES is applied in places where patients do exercises under the supervision of a specialist and involves the use of individual devices marketed to the general public to improve physical appearance. Many people exercise individually in such a way, especially their abdominal muscles. Abdominal muscles play an important role in lumbopelvic stability for optimizing performance. The effect of NMES training on abdominal muscles was examined in terms of muscle size, muscle strength, endurance, and lumbopelvic stability. A total of 23 subjects performed abdominal muscle NMES training for 8 weeks. Before and after the intervention, the cross-sectional area of the rectus abdominis muscle and lateral abdominal wall were measured by magnetic resonance imaging. In addition, muscle strength, endurance, and lumbopelvic stability were assessed. The study proved that NMES training resulted in a significant increase in all examined parameters [28].

An interesting electrostimulation solution was proposed by the authors of a study from Universidad Andrés Bello and Universidad Diego Portales, Santiago, Chile. They investigat- respondents' dominant hand. They reported a statistically significant increase in the gripping force $(p=0.0001)$ with the ascending current, i.e. the anode placed distally and cathode proximally. It was suggested that the findings could be of clinical interest and have applications in neurological or musculoskeletal disorders and in sports [29].

\section{Recommendations to be considered before applying electrical stimulation}

Before commencing electrical stimulation, it is necessary to pay attention to 2 issues. The first is thorough knowledge about alternating currents and their parameters that are the most important given the correct administration of muscle training or restoration of balance in neuromuscular excitability of paralyzed nerves and muscles. The second one is the apparatus: whether a given device has appropriate functions to select the detailed parameters, how many circuits can be used at once, and if it is possible to select different parameters for each circuit. It is necessary to remember that each muscle to which an electrode is applied has its physiological reaction to the applied frequency, as well as its strength and endurance. Therefore, identical doses of current should not be applied to different muscles and parts of the body. It should be emphasized that the correct selection of the current frequency is of great importance. It should depend on the aim of activities, i.e. whether one stimulates the contraction of healthy muscles or muscles with reduced neuromuscular excitability; whether one stimulates with or without the consideration of the pain reduction effect; whether one wants to increase blood circulation, additionally improve the lymph flow or whether it is not that important [30]. It often happens that therapists, people responsible for personal training in fitness clubs offering electrical stimulation of muscles in the training of their clients, are not aware of the significance of the correct frequency selection.

\section{Conclusions}

Electrical stimulation is a discipline in physical therapy which has been evolving for ages, since antiquity. The term is defined as any stimulation of the human body with low or medium frequency current. The correct application of currents for electrical stimulation requires a thorough knowledge about the condition of a muscle, selection of parameters corresponding with this condition, ability to operate the devices used in such procedures. An important aspect is to consider the risk of tissue destruction or rhabdomyolysis when excessive loads are applied. Therefore, training with electrical stimulation should be planned in compliance with methodology principles, with special attention paid to load limits.

\section{Ethical approval}

The conducted research is not related to either human or animal use.

\section{Disclosure statement}

The author does not have any financial interest and did not receive any financial benefit from this research.

\section{Conflict of interest}

The author states no conflict of interest.

\section{References}

1. Babault N, Cometti G, Bernardin M, Pousson M, Chatard J-C. Effects of electromyostimulation training on 
muscle strength and power of elite rugby players. J Strength CondRes.2007;21(2):431-437; doi:10.1519/R-19365.1.

2. Billot M, Martin A, Paizis C, Cometti C, Babault N. Effects of an electrostimulation training program on strength, jumping, and kicking capacities in soccer players. J Strength Cond Res. 2010;24(5):1407-1413; doi: 10.1519/ JSC.0b013e3181d43790.

3. Brocherie F, Babault N, Cometti G, Maffiuletti N, Chatard J-C. Electrostimulation training effects on the physical performance of ice hockey players. Med Sci Sports Exerc. 2005;37(3):455-460; doi: 10.1249/01.mss.0000 155396.51293.9f.

4. Watson T, Nussbaum EL (eds.). Electrophysical agents: evidence-based practice. Edinburgh: Elsevier; 2021.

5. Watson T. The role of electrotherapy in contemporary physiotherapy practice. Man Ther. 2000;5(3):132-141; doi: $10.1054 /$ math.2000.0363.

6. Low J, Reed A. Electrotherapy explained: principles and practice, $3^{\text {rd }}$ ed. Oxford: Butterworth Heinemann; 2000.

7. Chellappa D, Thirupathy M. Comparative efficacy of lowlevel laser and TENS in the symptomatic relief of temporomandibular joint disorders: a randomized clinical trial. Indian J Dent Res. 2020;31(1):42-47; doi: 10.4103/ ijdr.IJDR_735_18.

8. Heidland A, Fazeli G, Klassen A, Sebekova K, Hennemann $\mathrm{H}$, Bahner $\mathrm{U}$, et al. Neuromuscular electrostimulation techniques: historical aspects and current possibilities in treatment of pain and muscle waisting. Clin Nephrol. 2013;79(Suppl. 1):S12-S23; doi: 10.5414/ CNX77S106.

9. Lepley LK, Wojtys EM, Palmieri-Smith RM. Combination of eccentric exercise and neuromuscular electrical stimulation to improve quadriceps function post-ACL reconstruction. Knee. 2015;22(3):270-277; doi: 10.1016/ j.knee.2014.11.013.

10. Yao D, Jakubowitz E, Ettinger S, Claassen L, Plaass C, Stukenborg-Colsman C, et al. Functional electrostimulation for drop foot treatment: clinical outcome [in German]. Orthopade. 2017;46(3):227-233; doi: 10.1007/ s00132-016-3371-5.

11. Dehghani S, Rezaee A, Hosseinkhani S. Effect of alternating electrical current on denitrifying bacteria in a microbial electrochemical system: biofilm viability and ATP assessment. Environ Sci Pollut Res Int. 2018;25(33): 33591-33598; doi: 10.1007/s11356-018-3170-0.

12. De la Barra Ortiz HA, Opazo Cancino J, Peña FS, Soto León F, Miño Donoso E, Trecanao Gaete VT. Effectiveness of percutaneous microelectrolysis and ultrasound in decreasing pain in myofascial trigger points: evaluation through algometry and visual analogue scale. Physiother Quart. 2020;28(3):1-8; doi: 10.5114/PQ.2020. 95768.

13. Clinton S, Newell A, Downey PA, Ferreira K. Pelvic girdle pain in the antepartum population: physical therapy clinical practice guidelines linked to the International Classification of Functioning, Disability, and Health from the Section on Women's Health and the Orthopaedic Section of the American Physical Therapy Association. J Womens Health Phys Ther. 2017;41(2):102-125; doi: 10.1097/JWH.0000000000000081.

14. Tiktinsky R, Chen L, Narayan P. Electrotherapy: yesterday, today and tomorrow. Haemophilia. 2010;16(Suppl. 5): 126-131; doi: 10.1111/j.1365-2516.2010.02310.x.

15. Licht S. History of electrotherapy. In: Licht S (ed.), Therapeutic electricity and ultraviolet radiation, $2^{\text {nd }}$ ed. New Haven: E. Licht; 1967; 1-70.
16. Kramer JF, Mendryk SW. Electrical stimulation as a strength improvement technique: a review. J Orthop Sports Phys Ther. 1982;4(2):91-98; doi: 10.2519/jospt. 1982.4.2.91.

17. Mazur-Bialy Al, Kołomańska-Bogucka D, Nowakowski C, Tim S. Urinary incontinence in women: modern methods of physiotherapy as a support for surgical treatment or independent therapy. J Clin Med. 2020;9(4):1211; doi: 10.3390/jcm9041211.

18. Jha S, Walters SJ, Bortolami O, Dixon S, Alshreef A. Impact of pelvic floor muscle training on sexual function of women with urinary incontinence and a comparison of electrical stimulation versus standard treatment (IPSU trial): a randomised controlled trial. Physiotherapy. 2018; 104(1):91-97; doi: 10.1016/j.physio.2017.06.003.

19. Ma X-X, Liu A. Effectiveness of electrical stimulation combined with pelvic floor muscle training on postpartum urinary incontinence. Medicine. 2019;98(10):e14762; doi: 10.1097/MD0000000000014762.

20. Guo G-Y, Kang Y-G. Effectiveness of neuromuscular electrical stimulation therapy in patients with urinary incontinence after stroke: a randomized sham controlled trial. Medicine. 2018;97(52):e13702; doi: 10.1097/MD0 000000000013702.

21. Pastore AL, Palleschi G, Fuschi A, Al Salhi Y, Zucchi A, Bozzini $G$, et al. Pelvic muscle floor rehabilitation as a therapeutic option in lifelong premature ejaculation: long-term outcomes. Asian J Androl. 2018;20(6):572575; doi: 10.4103/aja.aja_30_18.

22. La Pera G. Awareness and timing of pelvic floor muscle contraction, pelvic exercises and rehabilitation of pelvic floor in lifelong premature ejaculation: 5 years experience. Arch Ital Urol Androl. 2014;86(2):123-125; doi: 10.4081/aiua.2014.2.123.

23. Laurienzo CE, Magnabosco WJ, Jabur F, Ferreira Faria E, Gameiro MO, Sari AJ, et al. Pelvic floor muscle training and electrical stimulation as rehabilitation after radical prostatectomy: a randomized controlled trial. J Phys Ther Sci. 2018;30(6):825-831; doi: 10.1589/jpts.30.825.

24. Kannan P, Winser SJ, Fung B, Cheing G. Effectiveness of pelvic floor muscle training alone and in combination with biofeedback, electrical stimulation, or both compared to control for urinary incontinence in men following prostatectomy: systematic review and meta-analysis. Phys Ther. 2018;98(11):932-945; doi: 10.1093/ptj/ pzy101.

25. Hauger AV, Reiman MP, Bjordal JM, Sheets C, Ledbetter L, Goode AP. Neuromuscular electrical stimulation is effective in strengthening the quadriceps muscle after anterior cruciate ligament surgery. Knee Surg Sports Traumatol Arthrosc. 2018;26(2):399-410; doi: 10.1007/ s00167-017-4669-5.

26. Lopes Evangelista AL, Vazquez La Scala Teixeira CV, Massaroto Barros B, Bezerra de Azevedo J, Paunksnis MRR, de Souza CR, et al. Does whole-body electrical muscle stimulation combined with strength training promote morphofunctional alterations? Clinics. 2019;74: e1334; doi: 10.6061/clinics/2019/e1334.

27. Stöllberger C, Finsterer J. Side effects of whole-body electro-myo-stimulation. Wien Med Wochenschr. 2019; 169(7-8):173-180; doi: 10.1007/s10354-018-0655-x.

28. Hwang U-J, Kwon O-Y, Jung S-H, Kim H-A, Gwak G-T. Effect of neuromuscular electrical stimulation training for abdominal muscles on change of muscle size, strength, endurance and lumbopelvic stability. J Sports Med Phys Fitness. 2020;60(2):206-213; doi: 10.23736/S0022-4707. 19.09998-5. 
29. De la Barra Ortiz HA, Opazo Cancino J, Minzer Goluboff N, Andrade Obando G, Herrera Jara M, González Vera MF. Effects of ascending and descending direct current on grip strength assessed through dynamometry and myofeedback: a randomized controlled trial. Physiother Quart. 2020;28(2):1-8; doi: 10.5114/pq.2020.92471.

30. Demidaś $A$, Zarzycki $M$. Touch and pain sensations in diadynamic current (DD) and transcutaneous electrical nerve stimulation (TENS): a randomized study. Biomed ResInt.2019;2019:9073073;doi:10.1155/2019/9073073. 